ORIGINAL ARTICLE

\title{
Bronchiectasis: Experience of Surgical Management at Tikur Anbessa Specialized Hospital, Addis Ababa, Ethiopia
}

\author{
Berhanu Nega $^{1}$, Yonas Ademe², AyalewTizazu ${ }^{1}$
}

\footnotetext{
OPEN ACCESS

Citation: Berhanu Nega, Yonas Ademe, AyalewTizazu. Bronchiectasis:

Experience of Surgical Management at Tikur Anbessa Specialized Hospital, Addis Ababa, Ethiopia. Ethiop J Health Sci.2017;29(4):471.doi:http://dx.doi.org/

10.4314/ejhs.v29i4.8

Received: March 4, 2019

Accepted: May 30, 2019

Published: July 1, 2019

Copyright: (C) 2019 Berhanu Nega, et al.

This is an open access article distributed under the terms of the Creative Commons

Attribution License, which permits unrestricted use, distribution, and reproduction in any medium, provided the original author and source are credited. Funding: Nil

Competing Interests: The authors declare that this manuscript was approved by all authors in its form and that no competing interest exists.

Affiliation and Correspondence:

${ }^{1}$ Cardiothoracic

Surgeon,Department of Surgery Unit of Cardiothoracic Surgery Addis Ababa University School of Medicine

${ }^{2}$ General Surgery Resident Department of Surgery Unit of Cardiothoracic Surgery Addis Ababa University School of Medicine

*Email: b_nega@yahoo.com
}

\section{ABSTRACT}

BACKGROUND: Bronchiectasis is one of the major health problems in Ethiopia. We analyzed the outcome of surgery done for bronchiectasis in a resource-limited setup.

METHODS: A retrospective cross-sectional analysis of 22 patients who underwent surgery for bronchiectasis in Tikur Anbessa specialized hospital (TASH) during the period 2012 - 2017 were done.

RESULTS: There were 13(59\%) female and 9(41\%) male patients with a mean age of $34.1+/-16.9$ years. The mean duration of symptoms was 2.2 years. Blood streak sputum, 13(59\%), dyspnea, 9(49.9\%), fetid sputum, 8(36.4\%), dry cough, 6(27.3\%), chest pain, 6(27.3\%) and massive hemoptysis 3(13.6\%) were the main presenting symptoms. In 20(91\%) of the patients, previous history of TB treatment was identified. Recurrent childhood infection, 1(4.5\%), and tumor obstruction, 1(4.5\%), were also seen. Bronchiectasis was left sided in 12(54.5\%), right-sided in 7(31.8\%) and bilateral in 3(13.6\%) patients. The disease affected multiple lung lobes in 9(40.9\%), left lower lobe in 6(27.3\%) and left upper lobe in $3(13.6 \%)$ cases. Indications for surgery were the failure of medical management in 10(45.5\%), destroyed lung in 9(40.9\%), and massive hemoptysis in $3(13.6 \%)$ cases. The procedures performed were lobectomy in $14(63.6 \%)$ and pneumonectomy in $8(36.4 \%)$ cases. Postoperative complications occurred in $5(22.7 \%)$ patients with one $(4.5 \%)$ death. On the other hand, $77.3 \%$ of operated patients had significant improvement compared to their preoperative symptoms.

CONCLUSIONS: In a resource-limited setup like TASH, localized bronchiectasis can be treated surgically with an acceptable result. Proper selection and preparation with complete resection of the involved segments are needed for maximum control of symptoms and better outcomes.

KEYWORDS: Bronchiectasis, Hemoptysis, Fetid sputum, Tuberculosis. 


\section{INTRODUCTION}

Bronchiectasis, originally described by René Laënnec in 1819 , is a suppurative lung disease characterized by permanent dilatations of bronchi with the destruction of the bronchial wall (1). Before the antibiotic era, the disease was considered a morbid disease with high mortality from respiratory failure and corpulmonale (2). The clinical picture differs greatly and may involve repeated respiratory infections with chronic production of sputum $(2,3,5)$. Today, with the improvement of healthcare and the availability of suitable antibiotics, the prevalence of bronchiectasis has declined (4). In developed countries, patients with early disease can be treated successfully by conservative procedures $(5,7)$. However, in developing countries like Ethiopia, bronchiectasis still constitutes an important problem because of tuberculosis, pneumonia, and pertussis (13). Current reports from developed countries about the surgical management for bronchiectasis show that limited localized disease was associated with good postoperative prognosis (2-11).

The objective of this study was to look into the surgical outcome of bronchiectasis in Tikur Anbessa Specialized Hospital (TASH) within a resource-limited situation. Its presentation, etiology, diagnostic tools, indications for surgery, surgical approach, and the outcome was analyzed.

\section{PATIENTS AND METHODS}

We retrospectively reviewed the medical records of 22 patients who underwent surgical resection for bronchiectasis between March 2012 and March 2017, at the Department of Cardiothoracic Surgery in Tikur Anbessa Specialized Hospital. Variables of age, sex, symptoms, etiology, involved lobe, indications and types of surgery, mortality, morbidity, and the results of surgical therapy were analyzed. Most patients were referred for surgery by their pulmonologist after long-term medical treatment, and some were admitted from our outpatient clinic as a referral from other hospitals. Preoperative assessment included clinical history, physical examination, Chest Radiography (CXR), computed tomography of the chest, and pulmonary function tests for selected patients.
Surgical treatment was considered when the symptoms persisted despite repeated courses of antibiotic treatment and if the extent of the diseased lung is localized to achieve complete resection. Surgery was also considered in cases with imaging features of destroyed lobe or lung. non-surgical treatments included the use of appropriate antibiotic, postural drainage, and bronchodilators. Symptoms that justify operations were recurrent pneumonia and/or recurrent hemoptysis.

A double-lumen endotracheal tube was used to minimize contralateral contamination of the lung with secretions. A posterolateral thoracotomy was used for all patients. Complete resection is defined as an anatomic resection of all affected segments that were assessed preoperatively by computed tomography. If the affected part of the lung is limited to one lobe, lobectomy was done and when the whole lung was affected, pneumonectomy was performed. Using a polypropylene suture in two layers, the bronchial stumps were closed.

Postoperative management included chest physiotherapy and administration of antibiotics and analgesics. Operative mortality included patients who died within 30 days after surgery or those who died later but during the same hospitalization.

All patients were followed up in our OPD. The clinical outcome of surgery was assessed by asking the patients to describe their current status in comparison to their preoperative condition. All data were stored using SPSS version 22.0 for Windows. Clinical data on different variables were reported as mean or ranges. When it is appropriate, the Chi-square test was used for comparisons.

\section{RESULTS}

A total of 22 patients who underwent surgical treatment for bronchiectasis in our department were included in this study. The mean age of these patients was $34.1 \pm 16.9$ years (range of 14 to 64 years). The female-to-male ratio was $13 / 9$ where $59.1 \%$ of the patients were female.

All patients were symptomatic. The most common presenting symptom was blood streak sputum which occurred in 13(59\%) of patients. 
Table 1: Sociodemographic charters of participants

\begin{tabular}{ll}
\hline Variables & No. (\%) \\
\hline Age (mean+/-SD) years & $34.1 \pm 16.9$ \\
Sex & \\
- Male & $9(40.9)$ \\
- Female & $13(59.1)$ \\
Symptoms & $8(36)$ \\
- Fetid sputum & $9(49.9)$ \\
- Shortness of breath & $6(27.3)$ \\
- Chest pain & $13(59)$ \\
- Blood streak sputum & $6(27.3)$ \\
- Dry cough & $3(13.6)$ \\
Causes of Bronchiectasis & $20(91)$ \\
- TB & $1(4.5)$ \\
- Recurrent childhood infection \\
- Tumor obstruction & $1(4.5)$ \\
Affected side & $12(54.5)$ \\
- Left & $7(31.8)$ \\
- Right & $3(13.6)$ \\
- Bilateral & \\
Affected Lobe & $6(27.3)$ \\
- Left lower lobe & $3(13.6)$ \\
- Left upper lobe & $2(9.1)$ \\
- Right lower lobe & $2(9.1)$ \\
- Right upper lobe & $9(40.9)$ \\
- Multilobar &
\end{tabular}

Copious amount of fetid sputum was found in $8(36.4 \%)$ patients, shortness of breath in $9(49.9 \%)$, and chest pain in $6(27.3 \%)$ patients. The mean duration of symptoms was 2.2 years (ranged of 6 months to 6 years). All patients had previous medical therapy and follow-up before admission.

Postero-anterior and lateral chest X-ray and CT scan were done for all patients. Diagnosis of bronchiectasis was based primarily on the chest CT scan finding. It determines the type and extent of bronchiectasis (Figure 1). The disease was on the left side in $12(54.5 \%)$ patients, in the right side in $7(31.8 \%)$, and bilateral in $3(13.6 \%)$ patients. Bronchiectasis involvement was multi-lobar in $9(40.9 \%)$ patients, predominantly in the lower lobes. The left lower lobe was affected in $6(27.3 \%)$ and the right lower lobe in $2(9.1 \%)$ patients (Table 1 ).

Table 1: Demographic characteristics, symptoms, causes, affected side and site of bronchiectasis.
The indications for pulmonary resection were the failure of medical therapy in $10(45.5 \%)$, destroyed lung in $9(40.9 \%)$, and recurrent or massive hemoptysis in $3(15.9 \%)$ cases. Posterolateral thoracotomy complete resections of all diseased segments were performed in all patients. Fourteen $(63.6 \%)$ patients had lobectomy and $8(36.4 \%)$ patients had pneumonectomy.

Complications occurred in 5(22.7\%) patients and included postoperative atelectasis requiring bronchoscopy in $1(4.5 \%)$ patient, pneumonia in $2(9.1 \%)$ patients, and bronchopleural fistula in $2(9.1 \%)$ patients. Operative mortality was seen in $1(4.5 \%)$ patients. The median hospital stay was 17 days (range of 8 to 54 days).

Follow-up data were obtained from all patients in the study. The symptoms disappeared in $17(77.3 \%)$ patients; $2(9.1 \%)$ patients showed residual symptoms, and $1(4.5 \%)$ patient died due to a postop medical complication.

Table 2: Indication, type and complication of surgery done for patients with Bronchiectasis.

\begin{tabular}{ll}
\hline Variables & No (\%) \\
\hline $\begin{array}{l}\text { Indications for surgery } \\
\text { Failure of medical }\end{array}$ & $10(45.5)$ \\
management & \\
Destroyed lung & $9(40.9)$ \\
Massive hemoptysis & $3(15.9)$ \\
Surgery done & \\
Lobectomy & $14(63.6)$ \\
Pneumonectomy & $8(36.4)$ \\
Complications & \\
Atelectasis & $1(4.5)$ \\
Pneumonia & $2(9.1)$ \\
Bronchopleural fistula & $2(9.1)$ \\
Death & $1(4.5)$ \\
\hline
\end{tabular}

DOI: http://dx.doi.org/10.4314/ejhs.v29i4.8 


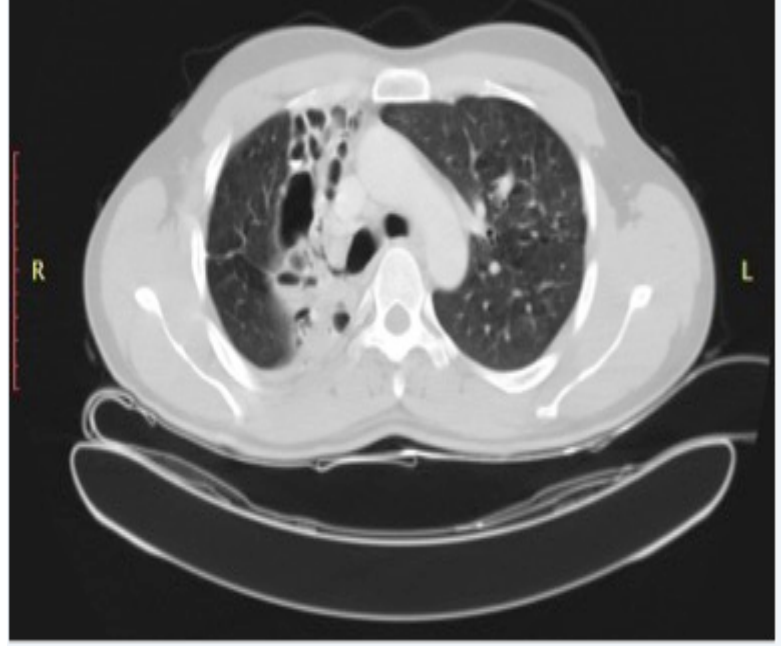

Figure 1: CT Scan of the chest that shows right upper lobe bronchiectasis

\section{DISCUSSION}

Bronchiectasis is pathologically defined as a condition in which there are abnormal and permanent dilatations of proximal bronchi (2). The cause for the bronchiectatic changes could be ending result of tuberculosis, bronchial obstruction due to sequestration, and foreign body or tumor (2-7). Before the antibiotic era, the disease was considered a morbid one with a high mortality rate from respiratory failure and corpulmonale $(7,19)$.

The incidence of bronchiectasis in Ethiopia is still unknown. However, from our clinical observation, it is still high. In developed countries, studies showed that its prevalence is currently decreasing because of the proper use of antimicrobial therapy and immunization against viral agents (2-10).

The optimal treatment for bronchiectasis still remains controversial (7). In cases of foreign body, early recognition and removal using Bronchoscope to minimize the subsequent development of bronchiectatic changes (6).

Different literatures reported different causes of bronchiectasis including Post TB, bronchial obstruction with either foreign body or tumor, immune deficiency, impaired mucocilinary clearance, inflammatory pneumonitis, structural airways abnormalities and association with other diseases (2-7). Other studies also reported that the cause is unknown in a significantly high percentage of patients ranging from $26 \%$ to $53 \%$ $(9,16)$.

Khaled et al (16) reported that predisposing factor was identified in only $35 \%$ of cases (predominately previous TB infection). In our study, post TB bronchial stenosis was the most common cause. All patients have history of treatment for pulmonary TB. They were all treated for 6 months to 6 years before they are admitted for surgery of bronchiectasis.

The typical presentation of patients with bronchiectasis is productive cough, recurrent pulmonary infections, bronchial suppuration, and purulent bronchorrhea (3). Similar to our series, blood streak sputum, cough, and fetid sputum are the most common symptoms in other series too (49). Out of 166 patients, Kutlay et al (9) reported that cough was reported in all patients; purulent sputum in 135, fetid sputum in 109 and hemoptysis was seen in 35 patients.

Bronchiectasis is basically diagnosed with a clinical history and CT scan findings. CT criteria for the diagnosis of bronchiectasis are well established which is an internal diameter of the bronchus more than 1.5 times than that of accompanying artery and evidence of lack of tapering of bronchi (11).

The most dependent portions of the lung that includes posterior basal portions of the lower lobes, middle lobe, and lingula are the primary sites for bronchiectasis. Generally, one-third of bronchiectasis is unilateral and affects a single lobe, one third is unilateral but affects more than one lobe, and one third is bilateral $(2,3)$. In our series, the disease affected the left lung in $12(54.5 \%)$ patients. It was mainly confined to the lower lobes either alone in $6(27.3 \%)$ or in conjunction with other lobes in $9(40.9 \%)$ patients.

Bronchiectasis should be treated initially with conservative management. Preventing recurrent infection, bronchodilator and chest physiotherapy (postural drainage) are the main components of conservative treatment. If conservative medical management is not successful or frequent episodes of hemoptysis exist, surgical therapy should be considered $(7,19)$. As was the case with other series, the indications for surgeries in our study were the failure of medical therapy, destroyed

DOI: http://dx.doi.org/10.4314/ejhs.v29i4.8 
lung, and recurrent hemoptysis in descending order (12-17).

The primary objectives of surgical intervention for bronchiectasis are to improve the quality of life, treat or prevent complications like empyema, recurrent hemoptysis, and lung abscess $(7,9,14)$. Because bronchiectasis is a progressive disease, the affected segment of the lung should be resected with an intention of preserving the uninvolved lung parenchyma. To achieve this goal, early surgery, while the disease is still localized, is preferred. In order to minimize cardiorespiratory failure, the aim of surgery should be complete resection with maximum preservation of lung function. Therefore, every type of resection is possible for these purposes. Ultimately, in order to ensure adequate lung function, a minimum of two lobes or six pulmonary segments must be spared. For successful surgery, Kutlay et al recommend that the operation should be performed in a 'dry period' (9).

Apart from the eight patients who underwent pneumonectomy for destroyed lung, most of our patients had a localized disease to one or more lobe. In selected patients with bilateral bronchiectasis, surgical therapy can be considered as one option of treatment. In our series, $2(9.1 \%)$ patients were operated on bilateral bronchiectasis.

Complication occurrence is $9.4-24.6 \%$ in the current literature. Therefore, our result of $(22.7 \%)$ is within the reported incidence (2-19). It included early and late complications such as prolonged air leak of more than 14 days, atelectasis, pneumonia, and bronchopleural fistula. Mortality ranges from $0 \%$ to $8.3 \%$ in the literature, and current mortality is less than $1 \%(2-20)$.

In our study, there was one $(4.5 \%)$ death. The patient was a 47-year-old male for whom the left upper lobectomy was done for an indication of post-tuberculosis bronchiectasis. The patient was doing well until postoperative day 3 when he started to have shortness of breath. He was found to be in respiratory distress with a respiratory rate of 35 breaths per minute, pulse rate of 108 beats per minute, and oxygen saturation of $65-70 \%$. There was decreased air entry over the lower twothirds of the left lung field. He had ECG and echocardiographic features of pulmonary embolism. The patient was transferred to the ICU the same day, and he was put on mechanical ventilator and anticoagulants. However, he passed away on a post-operative day 6 with immediate cause of death irespiratory failure secondary to massive pulmonary embolism.

In conclusion, even though the follow-up time was short, we did not compare the variation in exercise tolerance test and the changes in spirometry values; the overall patient assessment following surgery can be considered to have a satisfactory outcome. Similar with other studies, more than $77 \%$ of our the patients had a complete relief of their preoperative symptoms. For localized disease, with surgical treatment of bronchiectasis, our patients could obtain satisfactory results with acceptable morbidity and mortality.

Finally, we recommend that patients should continue their OPD follow-up visit for longer period, as assessment for recurrence requires many years of follow-up study. Proper patient selection and preparation with complete resection of the involved sites are needed for the optimum control of symptoms and better outcomes. As the number of cases operated on bronchiectasis is limited, awareness creation among physicians working in Ethiopia about the availability of surgical option for treatment of localized bronchiectasis specially for post TB need to be improved.

\section{ACKNOWLEDGMENT}

The authors would like to thank all the staff of the cardiothoracic surgery department at Tikur Anbessa Specialized Hospital for their assistance and support in conducting this research.

\section{REFERENCES}

1. Laënnec RTH. De l'Auscultation Médiate ou Trait du Diagnostic des Maladies des Poumon et du Coeur. Paris: Brosson \& Chaudé; 1819.

2. Paul T King; Review, The pathophysiology of bronchiectasis; International Journal of

COPD 2009:4 411-419

3. Kunter Balkanli, Onur Genc, Mehmet Dakak

DOI: http://dx.doi.org/10.4314/ejhs.v29i4.8 
et al; Surgical management of bronchiectasis: analysis and short-term results in 238 patients; European Journal of Cardio-thoracic Surgery; 2003; 24:699-702; doi: 10.1016/S1010-7940 (03) 00497-4

4. Riccardo Giovannetti, Marco Alifano, Alessandro Stefani; Surgical treatment of bronchiectasis: early and long-term results; Interactive CardioVascular and Thoracic Surgery; 2008; 7:609-612; doi: $10.1510 /$ icvts. 2007.163972

5. D. Coutinho, P. Fernandes, M. Guerra, J. Miranda, L. Vouga; Surgical treatment of bronchiectasis: A review of 20 years of experience; Rev Port Pneumol. 2016; 22(2): 82-85;

http://dx.doi.org/10.1016/j.rppnen.2015.09.00 7

6. Palasamudram shekar S, Bajarano $\mathrm{P}$, Hadeh A, et al.; Case of Missing Plastic: Foreign Body Bronchiectasis; Cureus; 2018; 10(7): 2974. DOI 10.7759/cureus. 2974

7. Vendrell M, de Gracia J, Olveira C, et al. Diagnosis and treatment of bronchiectasis. Spanish Society of Pneumology and Thoracic Surgery. Arch Bronconeumol 2008; 44:629-40

8. Prieto D, Bernardo J, Matos MJ, et al. Surgery for bronchiectasis. Eur J Cardiothorac Surg 2001;20:19-23.

9. Kutlay H, Cangir, Enön S, et al. Surgical treatment in bronchiectasis: analysis of 166 patients. Eur J Cardiothorac Surg 2002; 21:634-7.

10. Reda E. Al-Refaie, Sameh Amer, Mohamed El-Shabrawy, Surgical treatment of bronchiectasis: a retrospective observational study of 138 patients, J Thorac Dis; 2013; 5(3): 228-233; doi: 10.3978/j.issn.20721439.2013.04.11.

11. Naidich DP, McCauley DI, Khouri NF, Stitik FP, Siegelman SS; Computed tomography of bronchiectasis, J Comput Assist Tomogr. 1982 Jun; 6(3):437-44.

12. Giovannetti R, Alifano M, Stefani A, et al. Surgical treatment of bronchiectasis: early and long-term results. Interact Cardiovasc Thorac
Surg. 2008;7:609-12.

13. S. A. Adebonojo, O. Adebo, O. Osinowo; Experience with Bronchiectasis in Nigeria; Journal of the national medical association; 1979; 71(7): 687- 691.

14. Tomas Gudbjartsson ; Surgical resection is an effective treatment of bronchiectasis in selected patients; J Thorac Dis 2013; 5(3): 216. doi: 10.3978/j.issn.2072-1439.2013.06.21

15. Gokhan Haciibrahimoglu, Mithat Fazlioglu, Aysun Olcmen, Atilla Gurses, Mehmet Ali Bedirhan; Surgical management of childhood bronchiectasis due to infectious disease; The Journal of Thoracic and Cardiovascular Surgery; $\quad 2004 ; 127(5): 1361-1365$; doi:10.1016/j.jtcvs.2003.11.018.

16. Khaled M. Al-Kattan, Mohamed A. Essa, Waseem M. Hajjar, Mahmoud H. Ashour, Waleed N. Saleh, Mohamad A. Rafay, Surgical results for bronchiectasis based on hemodynamic (functional and morphologic) classification; $J$ Thorac Cardiovasc Surg; 2005; 130:1385-1390; doi: 10.1016/j.jtcvs.2005.06.026.

17. Sevval Eren, Hidir Esme, Alper Avci, Risk factors affecting outcome and morbidity in the surgical management of bronchiectasis; $J$ Thorac Cardiovasc Surg; 2007; 134: 392-8; doi: 10.1016/j.jtcvs.2007.04.024

18. Yu-xing Jin, Yi Zhang, Liang Duan, Yang Yang, Ge-ning Jiang, Jia-an Ding; Surgical treatment of bronchiectasis: A retrospective observational study of 260 patients; International Journal of Surgery; 2014; 12:1050-1054; doi.org/ 10.1016/j.ijsu.2014.08.398.

19. Miguel Ángel Martínez-García, Luis Máiz, Casilda Olveira, Spanish Guidelines on Treatment of Bronchiectasis in Adults; Arch Bronconeumol. 2018; 54(2):88-98.

20. Ufuk Cobanoglu, Irfan Yalcinkaya, Metin Er, et al; Surgery for Bronchiectasis: The effect of morphological types to prognosis; Ann Thorac Med;2011; 6(1):25-32; DOI: 10.4103/18171737.74273 . 